\title{
Circular Dichroism and Exciton Effect of Syndiotactic Methacrylic Polymers with Chiral Diacyl Hydrazide Side Chains V. Number of Exciton Coupled Monomeric Units
}

\author{
E. KLesper,${ }^{*}$ J. Thevissen, and D. KRIEGer \\ Lehrstuhl für Makromolekulare Chemie, Rheinisch-Westfälische \\ Technische Hochschule Aachen, Federal Republic of Germany
}

(Received August 29, 1987)

\begin{abstract}
Conformational order has been found in aqueous solution of tactic vinyl polymers derived from syndiotactic poly(methacrylic acid hydrazide). The CD spectra of poly(methacrylic acid- $N^{\beta}\left(\mathrm{L}\right.$-lactoyl)hydrazide) and poly(methacrylic acid- $N^{\beta}(N$-tert-butyloxycarbonyl-L-seryl) hydrazide) were studied in their deprotonated state, i.e., when containing diacyl hydrazide anion moieties. The experimental spectral traces were divided by a graphical subtraction procedure into an exciton doublet, representing monomeric units in conformational order, and Cotton effects due to monomeric units without such order. The resulting probabilities were used as a basis for a simulation procedure, carried out manually and by computer. The simulation yielded an estimate of the sequence statistics of ordered and unordered monomeric units in the polymer chain.
\end{abstract}

KEY WORDS Tacticity / Poly(methacrylic acid diacyl hydrazide) / Circular Dichroism / Exciton Effect / Conformational Order / Conformational Statistics / Simulation /

To obtain conformationally ordered vinyl polymers, one may start from a tactic base polymer which, by a polymer-analogous reaction, is transformed to a polymer capable of an ordered conformation. This route of preparation is preferable to a tactic polymerization of a corresponding monomer, if this monomer possesses functional groups which may interfere with ionic or Ziegler-Natta polymerization.

Previously, syndiotactic poly(methacrylic acid hydrazide) was prepared ${ }^{1,2}$ and reacted with different functional acids, e.g., $N$ protected L- $\alpha$-amino acids. ${ }^{1,3-6}$ The CDspectra indicated the presence of ordered conformations in aqueous solution by exhibiting high intensity Cotton and exciton effects, while the low molecular weight model compounds did not show such large effects. Exciton doublets were found in particular when the diacylhydrazide moiety was deprotonated, conferring polyelectrolyte character to the polymers, and when the side chain of the polymer contained an $-\mathrm{OH}$ or histidyl group. ${ }^{3-6}$

It is now of interest to determine the extent of the conformational order in the exciton coupled deprotonated polymers. Toward this end, the CD spectra are evaluated in this communication by means of a graphical subtraction procedure. The procedure allows to subdivide the experimental spectral trace into components which are due to conformationally ordered and to conformationally unordered monomeric units. Subsequently,

\footnotetext{
* To whom correspondence should be addressed.
} 
some simulations are carried out by an urn model, either by hand or by computer, to obtain a first estimate of the sequence statistics of the ordered and unordered monomeric units in the polymer chain.

\section{EXPERIMENTAL}

The syndiotactic homopolymers poly(methacrylic acid- $N^{\beta}$ (L-lactoyl) hydrazide) (L-sPMHL) $)^{1,3,4}$ and poly(methacrylic acid $-N^{\beta}(N$ tert-butyloxycarbonyl-L-seryl) hydrazide (L-s-PMHBocS), ${ }^{1,5}$ the cosyndiotactic copolymers (methacrylic acid hydrazide)-(methacrylic acid- $N^{\beta}$ (L-lactoyl) hydrazide) ((MH)-s$\mathrm{co}-(\mathrm{L}-\mathrm{MHL}))^{4}$ and (methacrylic acid hydrazide) -methacrylic acid- $N^{\alpha}(N$-tert-butyloxycarbonyl-L-seryl) hydrazide) ((MH)-s-co-(MHBocS) ${ }^{5}$ as well as the low molecular weight model compounds pivalic acid- $N^{\beta}$ (L-lactoyl)hydrazide (L-PivHL) ${ }^{4}$ and pivalic acid $-N^{\beta}(N$-tertbutyloxycarbonyl-L-seryl)hydrazide (L-PivHB$\mathrm{ocS})^{1,5}$ were prepared and characterized as previously described.

The CD spectra were obtained on a JobinYvon Dichrograph Mark IV in the concentration range of $0.1-1.0 \mathrm{mmoll}^{-1}$ in aqueous solution in fused quartz cuvettes with 1 $10 \mathrm{~mm}$ optical path length. The spectra were recorded at ambient temperature. For copolymers, the molar absorption coefficient difference, $\Delta \varepsilon$, was calculated on the basis of the molar concentration of the methacrylic acid- $N^{\beta}$ (L-lactoyl)hydrazide or the methacrylic acid- $N^{\beta}(N$-tert-butyloxycarbonyl-Lseryl)hydrazide monomeric units only, not considering the unacylated methacrylic acid hydrazide monomeric units. UV spectra were recorded on a Perkin-Elmer 554 UV/VIS spectrophotometer to yield an absorbance between 1.5 and 2.0. The corresponding concentration was then not to be exceeded for the CD spectra in order to obtain a good signal to noise ratio. The stability of the polymers in strongly alkaline solution was checked in several instances by observing the CD spectra over prolonged periods of time, or else by neutralizing the base after measurement and by ascertaining that the same CD and NMR spectra were obtained as in the beginning.

For the manual and the computer simulation of the sequence statistics of the coupled and uncoupled monomeric units, an urn model was employed. For the manual simulation, a set of 1000 monomeric units, consisting of the desired number of exciton coupled units (0) and uncoupled units, $(u)$, were randomly mixed in a vessel or urn and drawn one by one to form the simulated chain. The drawing was carried out with the special provision that a diad $(u 0)$ is necessarily followed by the monad $(0)$. That is, after drawing the sequence $(u 0)$, the drawing was continued until the triad $(u 00)$ was obtained, returning after each drawing all $(u)$ drawn in between, and randomizing thereby the content of the urn. This provision was necessary because éxciton coupling requires at least two adjacent monomeric units. After completing a triad ( $u 00)$, drawing was continued normally. After completion of the simulated chain containing 1000 monomeric units, the numbers of each of the six possible triads were counted.

A computer simulation by a Fortran program was carried out similarly to the manual operation, the difference being that 10 chains of 10.000 monomeric units each were simulated and the results of the 10 chains averaged. Moreover, the program provided for the presence of a third type of monomeric unit (c), i.e., the unacylated monomeric units in copolymers or the neutral acylated units in not fully deprotonated homopolymers. Two limits $G_{1}$ and $G_{2}$ were set as numbers between 0 and 1 and $0<G_{1}<G_{2} \leqslant 1$. A pseudorandom number between 0 and 1 was then generated, which, when falling between 0 and $G_{1}$ (inclusive), had the effect of drawing monad (0) from the initially 10.000 monomeric units in the urn. When falling between $G_{1}$ and $G_{2}$ (inclusive) it was monad ( $u$ ), while between $G_{2}$ and 1 (inclusive) it was monad (c) which 
was drawn. The provision that the sequence (u0) was necessarily to be followed by $(u 00)$ was upheld also for the computer program. After each drawing by a given random number, the limits $G_{1}$ and $G_{2}$ were recalculated according to the number $N$ of monads $(0),(u)$, and (c) then remaining in the urn, i.e., $G_{1}=N_{0} /\left(N_{0}+N_{u}+N_{c}\right) \quad$ and $\quad G_{2}=G_{1}+N_{u} /$ $\left(N_{0}+N_{u}+N_{c}\right)$, where the subscripts identify the kind of monad.

\section{RESULTS AND DISCUSSION}

Homo- and copolymers have been prepared, starting from syndiotactic poly(methacrylic acid hydrazide) (s-PMH). Thereby, the s-PMH was either reacted with L-lactic acid or with $N$-tert-butyloxycarbonyl-L-serine in water in the presence of $N$-ethyl- $N^{\prime}$ (3-dimethylamino propyl)carbodiimide hydrochloride (EAPC) to yield polymers with diacylhydrazide type side chains

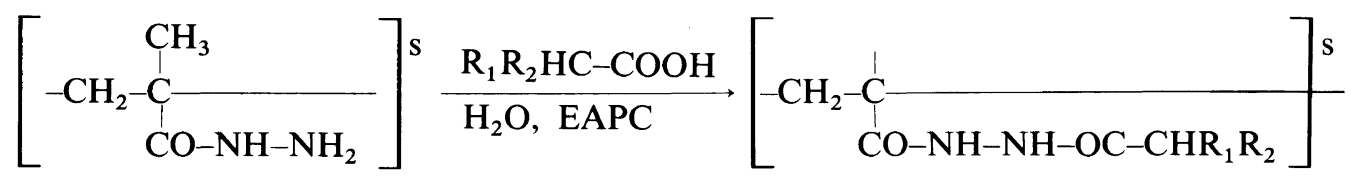

$$
\begin{aligned}
& \left.\begin{array}{l}
\mathrm{s}=\text { syndiotactic linking of repeat units } \\
\mathrm{R}_{1}=-\mathrm{CH}_{3} \\
\mathbf{R}_{2}=-\mathrm{OH}
\end{array}\right\} \text { L-lactic acid } \\
& \left.\begin{array}{l}
\mathrm{R}_{1}=-\mathrm{NHCOOC}\left(\mathrm{CH}_{3}\right)_{3} \\
\mathbf{R}_{2}=-\mathrm{CH}_{2} \mathrm{OH}
\end{array}\right\} N \text {-tert-butyloxycarbonyl-L-serine }
\end{aligned}
$$

By complete acylation, the syndiotactic homopolymers poly(methacrylic acid $-N^{\beta}(\mathrm{L}-$ lactoyl)hydrazide) (L-s-PMHL) and poly(methacrylic acid- $N^{\beta}(N$-tert-butyloxycarbonyl-L-seryl)hydrazide) (L-s-PMHBocS) were obtained and studied by CD spectroscopy in aqueous solution. Also studied by $\mathrm{CD}$ were syndiotactic copolymers obtained by a corresponding partial acylation of s-PMH, i.e., (methacylic acid hydrazide)-(methacrylic acid- $N^{\beta}$ (L-lactoyl)hydrazide) ((MH)-sco-(L-MHL)) and (methacrylic acid hydrazide) -(methacrylic acid- $N^{\beta}(N$-tert-butyloxycarbonyl-L-seryl)hydrazide) ((MH)-s-co-(L-MHBocS)). In order to draw conlusions about the conformational behavior of these polymers, model compounds were prepared from pivalic acid hydrazide (PivH), i.e., pivalic acid- $N^{\beta}$ (L-lactoyl)hydrazide (L-PivHL) and pivalic acid- $N^{\beta}(N$-tert-butyloxycarbonyl-Lseryl)hydrazide (L-PivHBocS).

All polymers show $\mathrm{CD}$ spectra in aqueous solution which are greatly dependent on $\mathrm{pH}$.
In Figure 1 the $C D$ spectra of the polymer L-sPMHL are shown at different $\mathrm{pH}$. Both in the acidic and in the strongly alkaline region pronounced CD effects are seen. Noteworthy is particularly the appearance of a doublet peak of opposite signs in the alkaline range, which grows strongly in size with increasing $\mathrm{pH}$. On the other hand, the model compound is much less sensitive to $\mathrm{pH}$ in the acidic range and the intensity remains at a much lower level. In the alkaline range, the difference in intensity between model and polymer is not quite as large, as seen when comparing Figure 2 to Figure 1. The intensity of the model also rises with $\mathrm{pH}$, but the model exhibits mainly a positive Cotton effect (Figure 2), while the polymer leads to the strong doublet peak.

The CD spectra of the polymer L-sPMHBocS in Figure 3 show a doublet peak in the alkaline region similar to L-s-PMHL, but with about twice the intensity and the order of signs reversed. The model compound LPivHBocS shows also a positive band which 


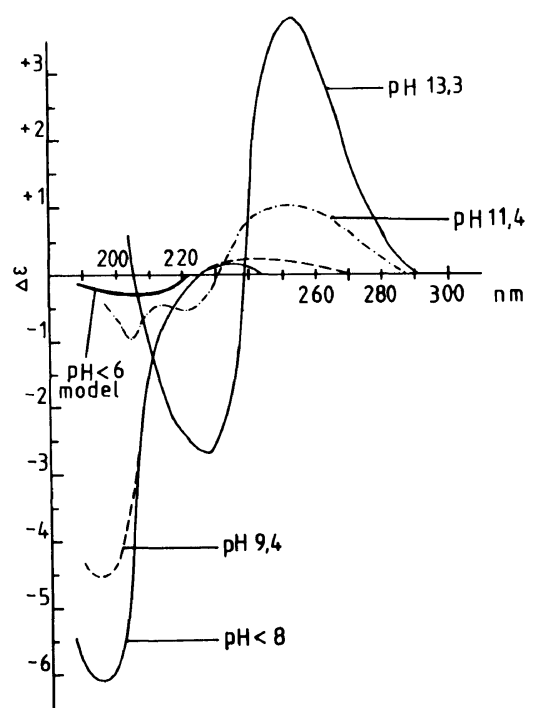

Figure 1. CD spectra of the polymer L-s-PMHL and its model compound L-PivHL in aqueous solution at different $\mathrm{pH}$.

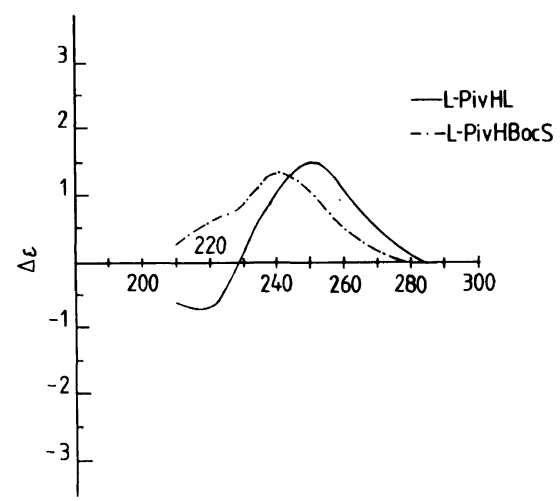

Figure 2. CD spectra of the model compounds LPivHL and L-PivHBocS in aqueous solution in the fully deprotonated state at alkaline $\mathrm{pH}$.

increases in intensity with rising $\mathrm{pH}$, but with much smaller intensity than the double band (Figure 2). The behavior of L-s-PMHBocS in the acidic region is different from L-S-PMHL in as much as only L-s-PMHL shows a much stronger Cotton effect than its model, while Ls-PMHBocS shows Cotton effects of small size, comparable in intensity and shape to its model L-PivHBocS.

The UV spectra of both polymers and both models show in the alkaline region a strong $\pi \rightarrow \pi^{*}$ absorption at $\lambda_{\max }=240-242 \mathrm{~nm}$ which is attributed to the diacyl hydrazide anion. The absorption is far removed from the $\pi \rightarrow \pi^{*}$ absorption of the two carbonamide groups in the undeprotonated diacylhydrazide group which is located below $200 \mathrm{~nm}$. The two carbonamide groups are transformed to one diacylhydrazide anion and vice versa, depending on $\mathrm{pH}$. It may be assumed that the diacylhydrazide anions have lost free rotation about their $\mathbf{N}-\mathbf{N}$ bond, which free rotation is present for the carbonamide groups. The anion may be regarded as a single chromophore in contrast to the two carbonamide chromophores in the neutral diacryl hydrazide moiety.

The CD spectra of the polymers in their diacyl hydrazide anion state have been interpreted as exciton doublet peaks which indicate conformational order in the polymers. $^{3-6}$ The side chains on neighboring monomer units containing the diacylhydrazide anions assume a fixed chiral orientation to each other. This leads to coupling of the electric dipole transition moments of the adjacent diacyl hydrazide anion chromophores. Since it can be assumed that the transition moment is in approximately the same direction as the long axis of the diacylhydrazide anion, the order of signs of the doublet peaks reflects the sense of spiralization in the relative orientation of the neighboring side chains. The order of signs for L-s-PMHL is $(+)(-)$, as counted from long to short wavelengths, while it is $(-)(+)$ for L-s-PMHBocS. According to exciton theory, there exists, therefore, a right handed spiralization for the first, and a left handed spiralization for the second polymer. ${ }^{7}$ A spiralization of this kind is possible, for instance, when the polymer backbone is in a $4_{2}$ helical conformation. ${ }^{4,5}$

The CD spectra of the deprotonated model compounds do not show an exciton doublet but mainly a positive Cotton effect which is located at or near the UV- $\lambda_{\max }$. The location is, however, in contrast to that of the exciton 
doublet peaks of the polymers, where it is the crossover point of the doublet which approximately coincides with the UV- $\lambda_{\max }$. The molecules of the model in solution are in random orientation and cannot possess a fixed spatial orientation towards each other whose life time is comparable to the fixed spatial orientation in the polymer on the CD time scale.

The presence of an exciton effect and of a corresponding conformational order is also made likely by the behavior of the doublet peak on the addition of salts or of alcohols. Both influence the conformational order, whereby the salt leads to a reduction of the intensity of the doublet peak (Figure 3) while lower amounts of alcohol may lead to an increase and higher amounts to a decrease in intensity. ${ }^{4,5}$ Strong support for the presence of exciton coupling comes, moreover, from the $\mathrm{CD}$ spectra of the copolymers $(\mathrm{MH})$-S- $\mathrm{Co}-(\mathrm{L}-$ $\mathrm{MHL}$ ) and (MH)-s-co-(L-MHBocS). In Figure 4 the CD spectra of (MH)-s-co-(L-MHL) in the deprotonated state at different copolymer composition, $P(A)$, is shown. $P(A)$ is the probability, which is numerically equal to the mol fraction, of monomeric units containing diacylhydrazide side chains, as determined by ${ }^{1} \mathrm{H}$ NMR. At $P(A)=0.2$, the diacylhydrazide anion monomeric units are surrounded predominantly by neutral monoacylhydrazide monomeric units as next neighbors along the chain, while at $P(A)=0.5$ and 0.8 the diacylhydrazide anion monomeric units are increasingly surrounded by like units. While diacylhydrazide anion units standing isolated on the chain are not able to undergo exciton coupling, the diacylhydrazide anion units surrounded by like units are able to couple, at least in principle. At $P(A)=1$ finally, i.e., with the homopolymer containing only L-MHL units and no $\mathrm{MH}$ units, all diacylhydrazide anion units must possess two adjacent diacylhydrazide anion neighbors. Therefore, at $P(A)=$ 0.2 , a normal Cotton effect is seen which is very similar to the Cotton effect of the model. With increasing $P(A)$, the doublet peak arises

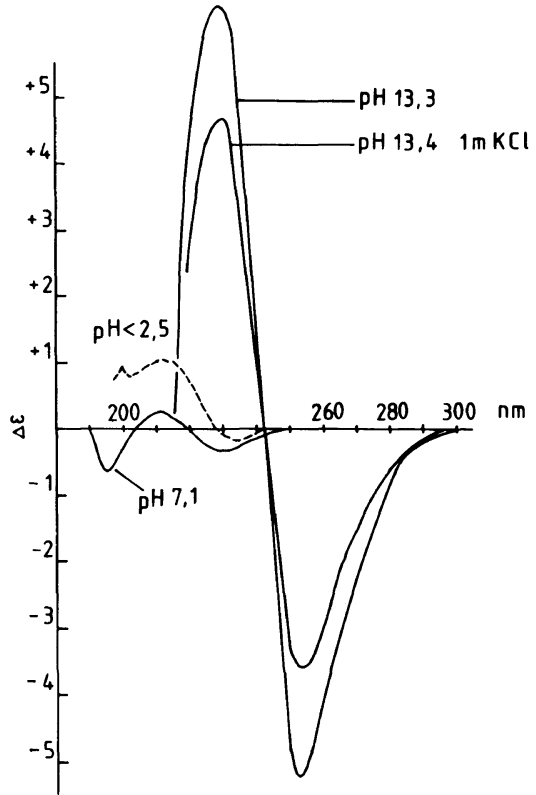

Figure 3. CD spectra of the polymer L-s-PMHBocS in aqueous solution at different $\mathrm{pH}$ and after addition of salt at $\mathrm{pH}=13.4$.
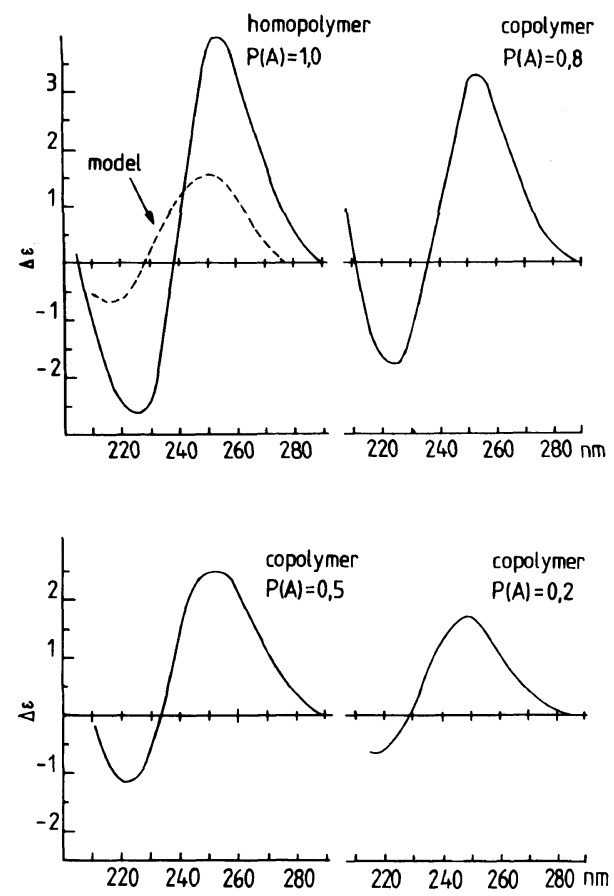

Figure 4. $\mathrm{CD}$ spectra of the copolymers $(\mathrm{MH})$-s-co-(LMHL) and the model compound L-PivHL in the deprotonated state at alkaline $\mathrm{pH} . P(A)$ is the probability (mol fraction) of finding a L-MHL monomeric unit. 

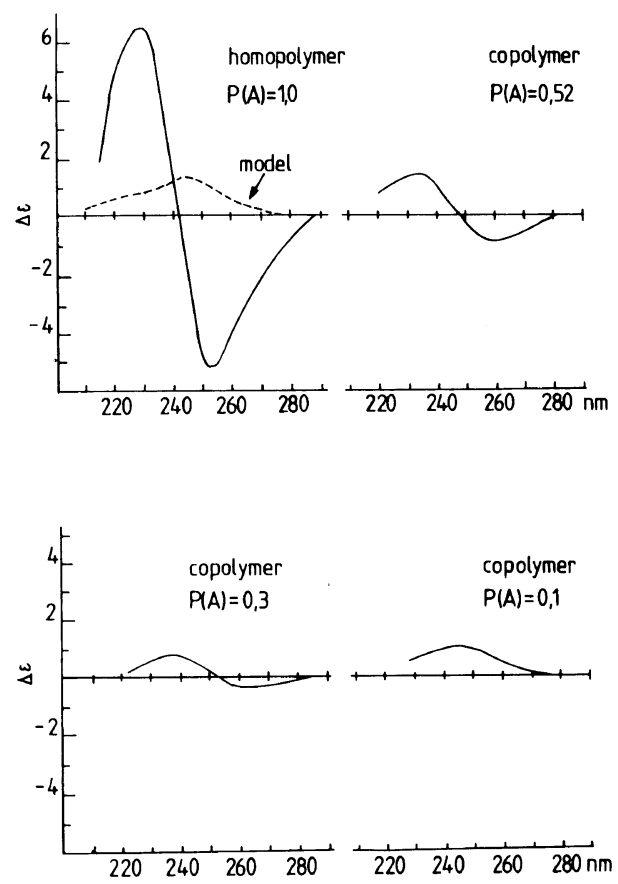

Figure 5. CD spectra of the copolymers (MH)-s-co-(LMHBocS) and the model L-PivHBocS in the deprotonated state at alkaline $\mathrm{pH}$. Copolymers of different compositions $P(A)$ ( $A=L$-MHBocS monomeric unit).

and reaches its maximum intensity at $P(A)=$ 1.0 .

The CD-spectra of the copolymers (MH)-sco-(L-MHBocS) in their deprotonated state are seen in Figure 5. Here $P(A)$ refers also to diacylhydrazide anion monomeric units, whereby, however, the L-lactoyl group is replaced by the tert-butyloxycarbonyl-L-seryl moiety. The appearance of the spectra and the development of the doublet peak is similar to Figure 4, although the intensity of the doublet peak is ultimately higher and the order of signs reversed. Thus, for both types of copolymers, a spatial proximity of chromophores is necessary for a doublet peak to arise.

According to exciton theory, ${ }^{7}$ the area of the two peaks of the doublet should be the same but of opposite sign. This, however, is not fully the case in Figures 1, 3, 4, and 5. For the
Table I. CD data of the homopolymer L-s-PMHL $(P(A)=1.0)$ and the $(\mathrm{MH})$-s-co-(L-MHL) copolymers of $P(A)=0.2,0.5$, and 0.8 . $\Delta \varepsilon$, $\lambda_{\max }$ and $\lambda_{0}$ (crossover) are given for the deprotonated state in the alkaline region in water at room temperature

\begin{tabular}{|c|c|c|c|c|c|}
\hline & $P(A)$ & $\mathrm{pH}$ & $\Delta \varepsilon$ & $\frac{\lambda_{\max }}{\mathrm{nm}}$ & $\frac{\lambda_{0}}{\mathrm{~nm}}$ \\
\hline$(\mathrm{MH})-\mathrm{s}-c o-(\mathrm{L}-\mathrm{MHL})$ & 0.2 & 13 & $\begin{array}{l}+1.75 \\
-0.75\end{array}$ & $\begin{array}{l}250 \\
219\end{array}$ & - \\
\hline$(\mathrm{MH})-\mathrm{s}-c o-(\mathrm{L}-\mathrm{MHL})$ & 0.5 & 13 & $\begin{array}{l}+2.5 \\
-1.15\end{array}$ & $\begin{array}{l}252 \\
223\end{array}$ & 234 \\
\hline (MH)-s-co-(L-MHL) & 0.8 & 13 & $\begin{array}{l}+3.3 \\
-1.75\end{array}$ & $\begin{array}{l}253 \\
225\end{array}$ & 236 \\
\hline L-S-PMHL & 1.0 & 13 & $\begin{array}{l}+4.0 \\
-2.6\end{array}$ & $\begin{array}{l}254 \\
225\end{array}$ & 238 \\
\hline L-PivHL & & 12.5 & $\begin{array}{l}+1.55 \\
-0.7\end{array}$ & $\begin{array}{l}250 \\
219\end{array}$ & 228 \\
\hline
\end{tabular}

homopolymer L-s-PMHL (Figures 1 and 4) the long wave length peak is too large, while for the homopolymer L-s-PMHBocS (Figures 3 and 5) the short wavelength peak appears to be somewhat larger. In either case, it is the positive component of the exciton doublet which is too large. The $\lambda_{\max }$ of the two peaks of an exciton doublet are expected, moreover, to be located at about equal distance from their crossover. There are also deviations from this requirement, particularly for L-s-PMHL. The data for $\Delta \varepsilon, \lambda_{\max }$, and $\lambda_{0}$ (crossover) for the homopolymer L-s-PMHL and the corresponding copolymers (MH)-s-co-(L-MHL) are given in Table I.

The requirements of equal area, about equal $\Delta \varepsilon$, and about equal distance of $\lambda_{\max }$ from the crossover $\lambda_{0}$ is more closely met, if it is realized that not all diacylhydrazide anions in the polymer are exciton coupled. The forces holding the side chains in a fixed chiral orientation to each other are counteracted by the thermal forces, leading to only partial orientation, e.g., part of the polymer is conformationally ordered and the other part is unordered. The ordered part results in an exciton doublet, whereas the unordered part leads to normal 
Cotton effects, as found in its pure form for the model compounds and for the copolymers of very low $P(A)$. The exciton doublet and the latter Cotton effects superimpose for the copolymers of higher $P(A)$ and the homopolymers. Because the simple Cotton effects are positive and located in the wave length range of the exciton doublet, the positive component of the exciton doublet always appears larger and the negative component smaller. In Figure 6, the exciton doublet of the polymer L-S-PMHL and the Cotton effects of the model L-PivHL are shown. Substraction of the single Cotton effect from the exciton doublet leads to a shape of the exciton doublet which appears to be more symmetrical with respect to area, $\Delta \varepsilon$, and shape.

As a second step, the spectral trace of the model L-PivHL is reduced in size by graphical reduction, using a constant factor $(<1)$ for reducing the heights at closely spaced wavelengths. The reduction is carried to the point, where, on subtraction of the reduced spectral trace of the model from the measured spectral trace of the polymer L-s-PMHL, an exciton effect is obtained for which the absolute magnitudes of the $\Delta \varepsilon$ for the positive and the negative component become equal. This is the case for L-PivHL and L-s-PMHL with a reduction factor of $\approx 0.80$. Accordingly, about $80 \%$ of the diacylhydrazide monomeric units are in a conformationally unordered state, while only $20 \%$ are ordered. The subtraction procedure also moves the crossover in the direction of the positive component of the exciton doublet. This is the same trend as seen in Table I for the copolymers when $P(A)$ rises. The crossover then corresponds more closely to $\lambda_{\max }=240-242$ of the UV spectra and the distances of the $\lambda_{\max }$ of the CD spectra from the crossover become more equal.

The percentages of the monomeric units in the ordered and unordered state can be changed in both polymers L-s-PMHL and L-S-PMHBocS, by changing the conditions, i.e., by adding salt or alcohol or by chang-

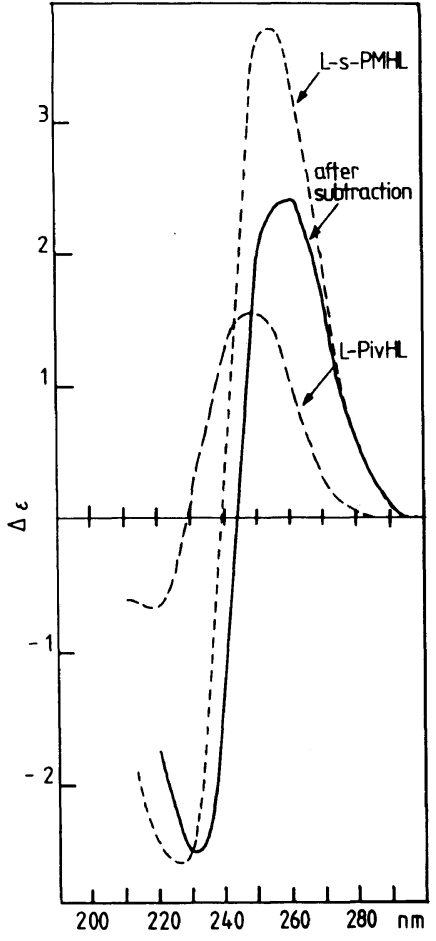

Figure 6. CD spectra of polymer L-s-PMHL and model L-PivHL in aqueous solution in the deprotonated state. The original spectral trace of the model is subtracted from that of the polymer to yield an exciton doublet of more equal magnitude in $\Delta \varepsilon$ for the two components of the doublet.

ing the temperature. ${ }^{4,5}$ Also, the substraction procedure should deal with integral intensities of CD bands rather than with $\Delta \varepsilon$. Such integral intensities are proportional to energy, if the CD-bands are plotted in units of $\Delta \varepsilon(\sigma) / \sigma$ on a wavenumber $(\sigma)$ scale. Thus, the more customary plot of $\Delta \varepsilon(\lambda) v s$. $\lambda$ in Figure 6 was used only as a first approximation. Using instead the plots $\Delta \varepsilon(\sigma) / \sigma$ for the subtraction procedure showed that the results of the subtraction may differ considerably between the two types of plots. In Figure 7 a plot of $\Delta \varepsilon(\sigma) / \sigma$ vs. $\sigma$ for the polymer L-SPMHL and its model compound L-PivHL is shown, both in the fully deprotonated state. Obviously, the low wave number peak of the $\pi \rightarrow \pi^{*}$ exciton doublet for L-s-PMHL is 


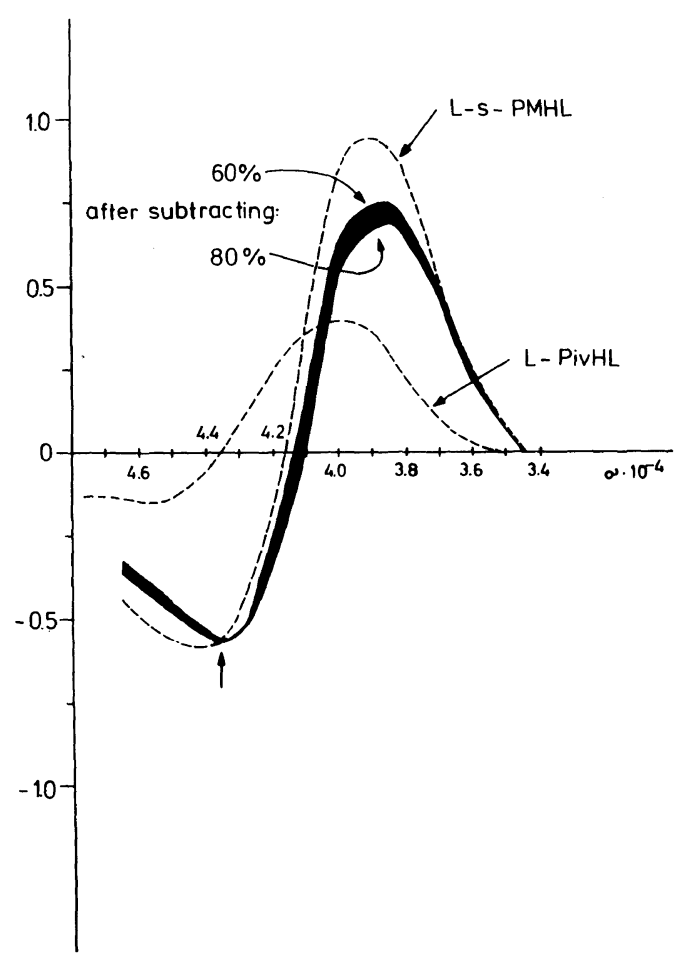

Figure 7. Plot of $\Delta \varepsilon(\sigma) / \sigma$ vs. $\sigma$ for the polymer L-sPMHL and its model compound L-PivHL in aqueous solution in the deprotonated state. The shown spectral trace of the model is then reduced to 80 or $60 \%$ of original height and the reduced traces are subtracted from the trace of L-s-PMHL. The resulting modified traces of L-S-PMHL are shown as the broad band whose border lines represent the reductions by 80 or $60 \%$. The arrow $(\uparrow)$ marks the point where the 80 and $60 \%$ lines cross over.

larger in area than the high wave number peak of the doublet. As before, this is caused by the superposition of the doublet with the Cotton effects of L-PivHL which represent the unordered conformation in L-SPMHL. There are at least two Cotton effects for L-PivHL which represent the unordered conformation in L-s-PMHL, a larger positive $\pi \rightarrow \pi^{*}$ transition at $40000 \mathrm{~cm}^{-1}$ and a smaller negative transition at about $45000 \mathrm{~cm}^{-1}$, the origin of the latter not being identified.

Reduction of the spectral trace of L-PivHL to 80 or $60 \%$ of its original height and subtracting the resulting trace from the spectral trace of L-s-PMHL leads to an exciton doublet whose two peaks are of more qual area, as demanded by theory. The percentage of reduction which is necessary to reach equal areas for the two peaks of the doublet of L-s-PMHL can only be approximately estimated. This is caused, first, by the missing high wave number part of the doublet. This part could in the present case not be measured on account of a high UV absorption at wave numbers higher than $46000 \mathrm{~cm}^{-1}$. Second, it cannot be excluded with certainty that there exist other CD bands for L-s-PMHL which superimpose with its exciton doublet. Third, on account of the relative positions of Frank-Condon potential curves for the ground and excited states, the low wave number peak of the doublet may be narrower with a steeper descend on its low wave number side than is the case for the high wave number peak. The latter peak is broader than the first. ${ }^{7}$ Taking these three factors into account, it may be estimated that the L-PivHL trace should be reduced to between 80 and $60 \%$ of its original height before subtraction, with 50 and $90 \%$ being the extremes. Nevertheless, the subtraction procedure not only leads to the conclusion that there exists a significant amount of ordered conformation but also that the amount of ordered conformation should not exceed $50 \%$ of all conformations and is probably between 20 and $40 \%$. This result is of interest in view of only a few methods available for the determination of the amount of ordered conformation in the dissolved state.

The substraction procedure itself rests on the assumption that the exciton effect of a given homopolymer depends only on the number of coupled units within a given type sequence distribution of coupled units. It does not depend on the assumption of additivity of the exciton effects of coupled units. Thus it is not required that the exciton effect of a tetrad of monomeric units is the sum of the exciton effects of the two diads contained in this tetrad. Instead, an exciton coupled monad is 
considered within a given, not a priory specified, type sequence distribution. Also, it is to be noted that the exciton doublet remaining after the subtraction procedure pertains to the ordered diacylhydrazide anion monomeric units only and not to all diacylhydrazide anions. The $\Delta \varepsilon$ of the ordered units, $\Delta \varepsilon_{0}$, is therefore obtained as

$$
\Delta \varepsilon_{0}=\Delta \varepsilon_{\mathrm{s}} \cdot \frac{100}{\%(0)}
$$

where $\Delta \varepsilon_{\mathrm{s}}$ is the apparent $\Delta \varepsilon$ after subtraction and $\%(0)$ is the percentage of ordered units.

The areas under the two peaks of the exciton doublet are related to the rotatory strength $R$ by

$$
R=2.296 \times 10^{-39} \int_{0}^{\infty} \frac{\Delta \varepsilon(\sigma)}{\sigma} \mathrm{d} \sigma
$$

whereby the integral is equal to the area of the peak in a plot of $\Delta \varepsilon(\sigma) / \sigma v s . \sigma$. The postulate of equal area for the positive $v s$. the negative peak of an exciton doublet may be expressed as

$$
\sum_{1}^{2} R_{n}=0 \quad n=1,2
$$

where equation 3 holds for the doublet after the subtraction procedure, i.e., for the apparent $\Delta \varepsilon(\sigma)$, i.e., $\Delta \varepsilon_{\mathrm{s}}(\sigma)$. Equation 3 holds also for the true $\Delta \varepsilon(\sigma)$, that is, for $\Delta \varepsilon_{0}(\sigma)$.

In Figure 8 the plot of $\Delta \varepsilon(\sigma) / \sigma v s . \sigma$ is given for the polymer L-S-PMHBocS and its model compound L-PivHBocS. Here, the low wave number peak is negative and the high wave number peak is positive. If unordered conformation would be present, the positive peak should be larger in area than the negative peak. This, however, is not the case. On the contrary, $R=1.12 \times 10^{-39}$ and $R=-1.22 \times$ $10^{-39}$ in cgs units, i.e., the positive peak is by $8 \%$ smaller in area, as based on the larger negative peak. Because the conformational order cannot exceed $100 \%$, the smaller area is apparently caused by partial overlap with a negative Cotton effect on the high wave num-

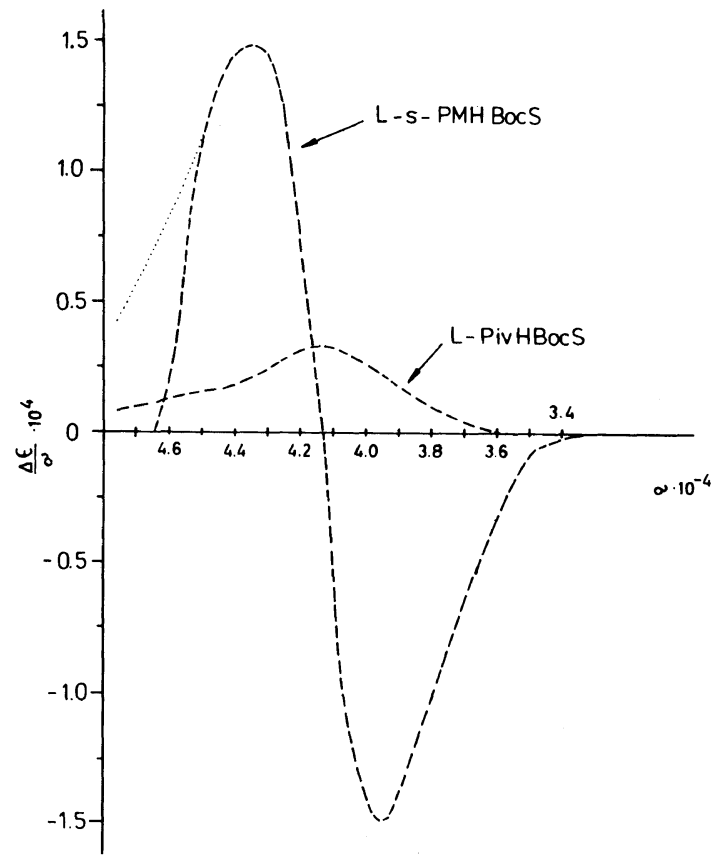

Figure 8. Plot of $\Delta \varepsilon(\sigma) / \sigma$ vs. $\sigma$ for the polymer L-SPMHBocS and model L-PivHBocS in aqueous solution in the deprotonated state. Experimental trace for L-sPMHBocS (-- ), assumed undisturbed trace for L-SPMHBocS $(\cdots)$. The spectral trace of the model is .reduced in size, until, on its subtraction from the assumed trace of the polymer, an exciton doublet with equal area of its peaks is obtained.

ber side. This overlap leads also to the observed steep descent on the high wave number side of the positive peak. Constructing an undisturbed positive peak for the doublet leads to an area for this peak which is probably not more than $20 \%$ larger than that of the negative peak. Carrying out the subtraction procedure then results in a still substantial amount of ordered conformation which should exceed $40 \%$ of all conformations.

The statistics of the ordered and unordered monomeric units along the polymer chain may be assumed to be random on account of the random nature of the thermal agitation, with the restriction that the minimum block length of ordered monomeric units is necessarily two. There must be at least two neighboring monomeric units present to define order, i.e., a fixed 
spatial orientation leading to exciton coupling. By an urn model which considers this restriction, and which is carried out manually, a chain of 1000 monomeric units is simulated. For simulating L-s-PMHL, 200 ordered and 800 unordered units are drawn at random one by one from the urn. The amounts of the two different units for L-s-PMHL corresponds to $P(0)=\%(0) / 100=0.20$ for the probability of ordered monomeric units and $P(u)=\%$ $(u) / 100=0.80$ for the unordered units. The urn model ( $c f$. Experimental) allows the formation of sequences of ordered units of length two or longer only. Counting the triads of monomeric units in the simulated chain results in an estimate of triad probabilities $P\left(X_{1} X_{2} X_{3}\right), X_{n}=0$ or $u$, given in Table II. The diad and monad probabilities were calculated by the principle of stationarity from the triad probabilities. For example,

$$
\begin{aligned}
P\left(0 u^{+}\right) & =P(0 u)+P(u 0) \\
P(0 u) & =P(0 u 0)+P(0 u u) \\
& =P(0 u 0)+0.5 P\left(0 u u^{+}\right) \\
P(u 0) & =P(u 00)+P(u 0 u) \\
& =0.5 P\left(00 u^{+}\right)+P(u 0 u)
\end{aligned}
$$

where the superscript + indicates the inclusion of both reverse forms, e.g., $0 u^{+}$includes $0 u$ and $u 0$.

The block lengths $n$, i.e., the average length of sequences of like monomeric units, are then obtained by

$$
\begin{aligned}
& \bar{n}(0)=\frac{P(0)}{0.5 P\left(0 u^{+}\right)} \\
& \bar{n}(u)=\frac{P(u)}{0.5 P\left(0 u^{+}\right)}
\end{aligned}
$$

which results in $\bar{n}(0)=2.2$ and $\bar{n}(u)=8.9$. Thus, on average only slightly more than two neighboring monomer units are in an ordered conformation leading to exciton coupling. Comparison of the simulated sequence statistics to ideal randomness of the distribution of
Table II. Estimate of the probabilities of conformational sequences of monomeric units in a manually simulated chain, based on $P(0)=0.20$ and $P(u)=0.80$

\begin{tabular}{lcll}
\hline Triads by $u r n^{\mathrm{a}}$ & $\begin{array}{c}\text { Triads } \\
\text { Bernoullic,d }^{\mathrm{c}}\end{array}$ & $\begin{array}{c}\text { Diads and } \\
\text { monads }^{\mathrm{a}, \mathrm{b}}\end{array}$ & $\begin{array}{c}\text { Diads } \\
\text { Bernoulli }^{\mathrm{c}}\end{array}$ \\
\hline$P(000)=0.013$ & $0.008(0.016)$ & $P(00)=0.104$ & 0.040 \\
$P(0 u 0)=0.013$ & $0.032(0.013)$ & $P\left(0 u^{+}\right)=0.181$ & 0.320 \\
$P\left(00 u^{+}\right)=0.183$ & $0.064(0.184)$ & $P(u u)=0.706$ & 0.640 \\
$P\left(0 u u^{+}\right)=0.155$ & $0.256(0.158)$ & $P(u)=0.797$ & - \\
$P(u 0 u)=0.000$ & $0.128(0.000)$ & $P(0)=0.196$ & - \\
$P(u u u)=0.629$ & $0.512(0.629)$ & & \\
\hline
\end{tabular}

a The + sign indicates reversal, e.g., $P\left(00 u^{+}\right)=$ $P(00 u)+P(u 00)$.

b Obtained by extension from triad probabilities.

c Obtained from $P(0)=0.20, P(u)=0.80$.

d Figures in parenthesis are obtained also by urn, but using a computer program ( $c f$. Experimental and text).

Table III. Estimate of the probabilities of conformational sequences of monomeric units in a computer simulated chain of $P(0)=0.40, P(u)=0.60$

\begin{tabular}{llll}
\hline Triads by $u r n^{\mathrm{a}}$ & $\begin{array}{c}\text { Triads } \\
\text { Bernoulli }^{\mathrm{c}}\end{array}$ & $\begin{array}{c}\text { Diads and } \\
\text { monads }^{\mathrm{a}, \mathrm{b}} \\
\text { by extension }\end{array}$ & $\begin{array}{c}\text { Diads } \\
\text { Bernoulli }^{\mathrm{c}}\end{array}$ \\
\hline$P(000)=0.075$ & 0.064 & $P(00)=0.237$ & 0.160 \\
$P(0 u 0)=0.050$ & 0.096 & $P\left(0 u^{+}\right)=0.324$ & 0.480 \\
$P\left(00 u^{+}\right)=0.324$ & 0.192 & $P(u u)=0.437$ & 0.360 \\
$P\left(0 u u^{+}\right)=0.225$ & 0.288 & & \\
$P(u 0 u)=0.000$ & 0.144 & $P(u)=0.600$ & \\
$P(u u u)=0.325$ & 0.216 & $P(0)=0.399$ & \\
\hline a The + sign indicates & reversal, e.g., & $P\left(0 u u^{+}\right)=$ \\
$P(0 u u)+P(u u 0)$. & & \\
b Obtained by extension from triad probabilities. \\
c Obtained from $P(0)=0.40, P(u)=0.60$.
\end{tabular}

0 and $u$ units, i.e., a Bernoullian statistics, shows the expected clustering to blocks of 0 units, a feature which is inherent in the urn model employed.

Performing the urn model by a Monte Carlo computer program and using $P(0)=0.40$, $P(u)=0.60$, as also compatible for the subtraction procedure for the polymer L-s-PMHL, the statistics of Table III was obtained. While the estimates of the triad probabilities in Table III are more accurate than those in Table II, a comparison between the manually and the 
computer obtained data shows no large discrepancy ( $c f$. Table II). Using the data in Table III for calculating the average block lengths yields $\bar{n}(0)=2.5$ and $\bar{n}(u)=3.7$. Thus the length of the blocks of ordered monomeric units is longer with a higher $P(0)$, as expected.

Acknowledgement. Financial support by the Deutsche Forschungsgemeinschaft is gratefully acknowledged.

\section{REFERENCES}

1. R. Vartan-Boghossian, B. Dederichs, and E. Klesper, Eur. Polym. J., 22, 23 (1986).

2. J. Thevissen, L. Klein, B. Dederichs, and E. Klesper, Eur. Polym. J., 24, 201 (1988).

3. J. Thevissen and E. Klesper, Polym. Commun., 28, 62 (1987).

4. J. Thevissen, E. B. Mano, and E. Klesper, J. Polym. Sci. A, Polym. Chem., in press.

5. J. Thevissen, E. B. Mano, and E. Klesper, Polymer, submitted for publication.

6. J. Thevissen and E. Klesper, Eur. Polym. J., submitted for publication.

7. N. Harada and K. Nakanishi, "Circular Dichroic Spectroscopy-Exciton Coupling in Organic Stereochemistry," University Science Books, Oxford University Press, 1983. 